Erratum

\title{
Erratum: Andreotti, S.; Franzoni, E.; Fabbri, P. Poly(hydroxyalkanoate)s-Based Hydrophobic Coatings for the Protection of Stone in Cultural Heritage. Materials 2018, 11, 165
}

\author{
Serena Andreotti ${ }^{1,2}$, Elisa Franzoni 1,2,*(D), Micaela Degli Esposti ${ }^{1,2}$ (D) and Paola Fabbri ${ }^{1,2}$ \\ 1 Department of Civil, Chemical, Environmental and Materials Engineering, University of Bologna, \\ 40131 Bologna, Italy; serena.andreotti2@unibo.it (S.A.); micaela.degliesposti@unibo.it (M.D.E.); \\ p.fabbri@unibo.it (P.F.) \\ 2 Consorzio Interuniversitario di Scienza e Tecnologia dei Materiali (INSTM), 50121 Firenze, Italy \\ * Correspondence: elisa.franzoni@unibo.it; Tel.: +39-051-209-0339
}

Received: 28 February 2018; Accepted: 2 March 2018; Published: 7 March 2018

The authors wish to add a new author, Micaela Degli Esposti, who also contributed to performing the experiments and analyzing the data of this published paper [1]. The correct authorship is shown below:

\footnotetext{
Serena Andreotti ${ }^{1,2}$, Elisa Franzoni ${ }^{1,2, *}$, Micaela Degli Esposti ${ }^{1,2}$ and Paola Fabbri ${ }^{1,2}$

1 Department of Civil, Chemical, Environmental and Materials Engineering, University of Bologna, 40131 Bologna, Italy; serena.andreotti2@unibo.it (S.A.); micaela.degliesposti@unibo.it (M.D.E.); p.fabbri@unibo.it (P.F.)

2 Consorzio Interuniversitario di Scienza e Tecnologia dei Materiali (INSTM), 50121 Firenze, Italy

* Correspondence: elisa.franzoni@unibo.it; Tel.: +39-051-209-0339
}

Author Contributions: Elisa Franzoni and Paola Fabbri conceived the study and designed the experiments; Serena Andreotti and Micaela Degli Esposti performed the experiments; Serena Andreotti, Elisa Franzoni, Micaela Degli Esposti and Paola Fabbri analyzed the data; Serena Andreotti and Elisa Franzoni collaborated on the writing of the paper; Paola Fabbri revised the paper. The authors are responsible for these errors, they regret any inconvenience or misunderstanding caused by them.

Conflicts of Interest: The authors declare no conflict of interest.

\section{Reference}

1. Andreotti, S.; Franzoni, E.; Degli Esposti, M.; Fabbri, P. Poly(hydroxyalkanoate)s-Based Hydrophobic Coatings for the Protection of Stone in Cultural Heritage. Materials 2018, 11, 165. [CrossRef] [PubMed]

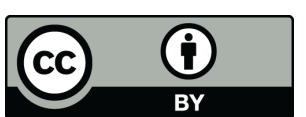

() 2018 by the authors. Licensee MDPI, Basel, Switzerland. This article is an open access article distributed under the terms and conditions of the Creative Commons Attribution (CC BY) license (http://creativecommons.org/licenses/by/4.0/). 